VOL. $4(1971), 289-305$.

\title{
Self adjoint operators and matrix measures
}

\section{Patrick J. Browne}

\begin{abstract}
Given a self adjoint operator, $T$, on a Hilbert space $H$, and given an integer $n \geq 1$, we produce a collection $\left(\mu_{i j}^{N}\right)$, $N \in L$, of $n \times n$ positive matrix measures and a unitary map $U: H \rightarrow \sum_{N \in L} L^{2}\left(\mu_{i j}^{N}\right)$ such that $U T U^{-1}$, restricted to the co-ordinate space $L^{2}\left(\mu_{i j}^{N}\right)$, is the multiplication operator $F(t) \rightarrow t F(t)$ in that space. This is a generalization of the spectral representation theory of Dunford and Schwartz, Linear operators, II (1966).
\end{abstract}

1.

In [1, XII.3], Dunford and Schwartz present a theory of spectral representation for self adjoint operators on a Hilbert space. The basic operator used for this representation can be described as follows. Consider a totally finite Lebesgue-Stieltjes measure $\mu$ on the real line and define an operator $A$ on $L^{2}(\mu)$ by

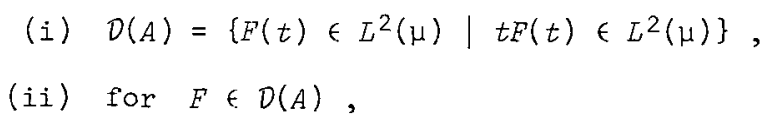

$$
(A F)(t)=t F(t)
$$

Received 16 November 1970. This paper forms part of the author's Ph.D. thesis submitted to The Flinders University of South Australia; the work was carried out under the supervision of Professor D.B. Sears while the author held a CSIRO Post-Graduate Studentsinip. 
It is well known that $A$ is self adjoint. The basic theorem then states that given a Hilbert space $H$ and a self adjoint operator $T$ on it, one can produce a collection of totally finite Lebesgue-Stieltjes measures $\mu_{\alpha}$ and a unitary map $U: H \rightarrow \sum_{\alpha} L^{2}\left(\mu_{\alpha}\right)$, such that for every Borel function $k$ defined on the spectrum of $T$,

$$
U D(k(T))=\left\{\left.F \in \sum_{\alpha} L^{2}\left(\mu_{\alpha}\right)\left|\sum_{\alpha} \int\right| k(t) F_{\alpha}(t)\right|^{2} d \mu_{\alpha}(t)<\infty\right\},
$$

and

$$
(U k(T) f)_{\alpha}(t)=k(t)(U f)_{\alpha}(t)
$$

The unitary map $U$ is then called a spectral representation of $H$ onto $\sum_{\alpha} L^{2}\left(\mu_{\alpha}\right)$ relative to $T$.

When the collection of measures $\mu_{\alpha}$ has cardinality 1 the operator $T$ is said to have simple spectrum. In this case, $T$ may be regarded as a unitary copy of the operator $A$ described above. Well-known examples are the self adjoint operators obtained from the Sturm-Liouville problem over a finite interval or half line.

It can be seen that this representation is centred on the choice of $A$ as the basic operator. The purpose of this paper is to discuss a corresponding theory when $A$ is replaced by the operator $B$ which may be described as follows. Consider an $n \times n$ positive matrix measure $\left(\mu_{i j}\right)$ on the real line. For $F=\left(F_{1}, F_{2}, \ldots, F_{n}\right) \in L^{2}\left(\mu_{i j}\right)$ and $k$ a Borel function defined on the line, let $k(t) F(t)=\left(k(t) F_{1}(t), \ldots, k(t) F_{n}(t)\right)$. Define $B$ by

$$
\begin{aligned}
& \text { (i) } D(B)=\left\{F(t) \in L^{2}\left(\mu_{i j}\right) \mid t F(t) \in L^{2}\left(\mu_{i j}\right)\right\} \text {, } \\
& \text { (ii) for } F \in D(B),
\end{aligned}
$$

$$
(B F)(t)=t F(t) .
$$

$B$ is known to be self adjoint. Such a representation theory will cover that discussed before - it can be obtained by taking $n=1$ in this 
theory.

The theory of positive matrix measures is discussed fully in [1, pp. 1337-1350].

2.

Let $H$ be a Hilbert space and $T: D(T) \subset H \rightarrow H$ a self adjoint operator with resolution of the identity $E$. For a finite set $N \subset H$, $N=\left\{f_{1}, f_{2}, \ldots, f_{n}\right\}, H_{N}$ will denote the closure of the manifold in $H$ consisting of all vectors of the form $k_{I}(T) f_{1}+\ldots+k_{n}(T) f_{n}$ where $k_{i}$ varies over all Borel measurable functions for which $f_{i} \in D\left(k_{i}(T)\right)$.

LEMMA 1. Let the complex valued measures $\mu_{i j}, 1 \leq i, j \leq n$, be defined by $\mu_{i j}(\cdot)=\left(E(\cdot) f_{i}, f_{j}\right)$. Then $\mu_{i j}$ is an $n \times n$ positive matrix measure on the Borel subsets of the real line, $R$.

Let $M$ be a Borel subset of $R$. Then

$$
\mu_{i j}(M)=\left(E(M) f_{i}, f_{j}\right)=\left(f_{i}, E(M) f_{j}\right)=\overline{\mu_{j i}(M)} .
$$

Thus $\left(\mu_{i j}(M)\right)$ is an Hermitian matrix. From the spectral theorem we know that each $\mu_{i j}$ is o-additive. Let $\xi_{1}, \ldots, \xi_{n}$ be complex numbers.

Then

$$
\begin{aligned}
\sum_{i, j=1}^{n} \mu_{i j}(M) \xi_{i} \bar{\xi}_{j} & =\sum_{i, j=1}^{n}\left(E(M) \hat{f}_{i}, f_{j}\right) \xi_{i} \bar{\xi}_{j} \\
& =\left(E(M) \sum_{i=1}^{n} \xi_{i} f_{i}, \sum_{j=1}^{n} \xi_{j} f_{j}\right) \geq 0,
\end{aligned}
$$

showing that $\mu_{i j}(M)$ is positive semi-definite. Thus $\left(\mu_{i j}\right)$ is an $n \times n$ positive matrix measure. Note that $\mu_{i j}$ is defined on all Borel subsets of $R$ and

$$
\left|\mu_{i j}(M)\right|=\left|\left(E(M) f_{i}, f_{j}\right)\right| \leq\left\|f_{i}\right\|\left\|f_{j}\right\| .
$$

LEMMA 2. Let $\left(\mu_{i j}\right)$ be as above. Define $\mu$ by 
$\mu(\cdot)=\sum_{i=1}^{n} \mu_{i i}(\cdot)$. Then $\mu$ is a totally finite measure on the Borel subsets of $R$ and each $\mu_{i j}$ is continuous with respect to $\mu$. Further, $H_{N}$ is a Hilbert space which is unitarily equivalent to $L^{2}\left(\mu_{i j}\right)$.

It is obvious that $\mu$ is a totally finite measure. Suppose $\mu(M)=0$, then $\mu_{i i}(M)=0$ for each $i=1,2, \ldots, n$. Further

$$
\begin{aligned}
\left|\mu_{i j}(M)\right| & =\left|\left(E(M) f_{i}, f_{j}\right)\right| \leq\left\|E(M) f_{i}\right\|\left\|f_{j}\right\| \\
& =\left(E(M) f_{i}, f_{i}\right)\left\|f_{j}\right\|=0 .
\end{aligned}
$$

Thus $\mu_{i j}(M)=0$ and so each $\mu_{i j}$ is continuous with respect to $\mu$. Note that the existence of such a measure $\mu$ enables us to construct the space $L^{2}\left(\mu_{i j}\right)$. Let $\left(m_{i j}\right)$ be the matrix of densities defined by the equations

$$
\mu_{i j}(M)=\int_{M} m_{i j}(t) d \mu(t), \quad 1 \leq i, j \leq n
$$

where $M$ is any Borel set.

It is clear that $H_{N}$ is a Hilbert space, being a closed linear manifold in $H$. Let $k_{1}(T) f_{I}+\ldots+k_{n}(T) f_{n} \in H_{N}$ and define

$$
\begin{aligned}
& k_{i}^{m}(t)=k_{i}(t) \text { if }\left|k_{i}(t)\right| \leq m \text {, } \\
& =0 \text { if }\left|k_{i}(t)\right|>m, m=1,2, \ldots, \text {. }
\end{aligned}
$$

Then each $k_{i}^{m}(T)$ exists as a bounded everywhere defined operator on $H$ and

$$
\sum_{i=1}^{n} k_{i}(T) f_{i}=\lim _{m \rightarrow \infty} \sum_{i=1}^{n} k_{i}^{m}(T) f_{i} .
$$

Thus 


$$
\begin{aligned}
\left\|\sum_{i=1}^{n} k_{i}(T) f_{i}\right\|^{2} & =\lim _{m \rightarrow \infty}\left(\sum_{i=1}^{n} k_{i}^{m}(T) f_{i}, \sum_{j=1}^{m} k_{j}^{m}(T) f_{j}\right) \\
& =\lim _{m \rightarrow \infty} \sum_{i, j=1}^{n}\left(k_{j}^{m}(T) * k_{i}^{m}(T) f_{i}, f_{j}\right\} \\
& =\lim _{m \rightarrow \infty} \sum_{i, j=1}^{n} \int_{-\infty}^{\infty} k_{i}^{m}(t) \overline{k_{j}^{m}(t)} d \mu_{i j}(t) \\
& =\lim _{m \rightarrow \infty} \int_{-\infty}^{\infty} \sum_{i, j=1}^{n} m_{i j}(t) k_{i}^{m}(t) \overline{k_{j}^{m}(t)} d \mu(t) \\
& =\int_{-\infty}^{\infty}\left\{\sum_{i, j=1}^{n} m_{i j}(t) k_{i}(t) \overline{k_{j}(t)}\right\} d \mu(t),
\end{aligned}
$$

since $\sum_{i, j=1}^{n} m_{i j}(t) k_{i}^{m}(t) \overline{k_{j}^{m}(t)}$ is a monotone increasing sequence of non-negative Borel functions tending pointwise to $\sum_{i, j=1}^{n} m_{i j}(t) k_{i}(t) \overline{k_{j}(t)}$. Thus $\left(k_{1}, \ldots, k_{n}\right) \in L^{2}\left(\mu_{i j}\right)$ and

$$
\left\|\sum_{i=1}^{n} k_{i}(T) f_{i}\right\|_{H_{N}}=\left\|\left(k_{1}, \ldots, k_{n}\right)\right\|_{L^{2}\left(\mu_{i j}\right)} \text {. }
$$

Using the correspondence $\sum_{i=1}^{n} k_{i}(T) f_{i} \leftrightarrow\left(k_{1}, k_{2}, \ldots, k_{n}\right)$ we can now set up a norm preserving linear map of a dense subset of $H_{N}$ into $L^{2}\left(\mu_{i_{i}}\right)$, which can therefore be extended to an isometry of $H_{N}$ into $L^{2}\left(\mu_{i j}\right)$. Now let $\left(k_{1}, k_{2}, \ldots, k_{n}\right) \in L^{2}\left(\mu_{i j}\right)$. Then defining $k_{i}^{m}$ as before we see from the above argument that $\sum_{i=1}^{n} k_{i}^{m}(T) f_{i} \in H_{N}$ and $\left\|\left(k_{1}^{m}, \ldots, k_{n}^{m}\right)\right\|=\left\|\sum_{i=1}^{n} k_{i}^{m}(T) f_{i}\right\|$. Taking the limit as $m \rightarrow \infty$ we deduce the existence of a point $f \in H_{N}$ such that $i\left(k_{I}, \ldots, k_{n}\right)\|=\| f \|$. This shows that the isometry produced in the first half of the proof is onto 
and so the spaces $H_{N}$ and $L^{2}\left(\mu_{i j}\right)$ are unitarily equivalent.

LEMMA 3. Let $n \geq 1$ be an integer. Then there is a collection $L$ of finite subsets $N \subset H$, each containing $n$ points, such that

$$
H=\sum_{N \in L} H_{i V} .
$$

Consider the family $K$ of collections $\lambda$ of finite subsets $N \subset H$ each containing $n$ points and such that the spaces $H_{N}, N \in \lambda$, are mutually orthogonal. Order $K$ by inclusion. Let $\Lambda$ be a chain in $K$ and put $E=\underset{\lambda \in \Lambda}{U} \lambda$. Then $E$ is a collection of finite subsets $N \subset H$ each containing $n$ points. Further, if $N_{1}, N_{2} \in E$, there exists $\lambda \in \Lambda$ such that $N_{1}, N_{2} \in \lambda$ and so $H_{N_{1}} \perp H_{H_{2}}$. We may now apply Zorn's Lemma to deduce the existence of a maximal collection $L$ of finite subsets $N \subset H$ each containing $n$ points such that the spaces $H_{N}, N \in L$, are mutually orthogonal. Thus to prove the result, it suffices to show that no $f \neq 0$ is orthogonal to each of the spaces $H_{N}, N \in L$. If there exists $f \neq 0$ such that $f \perp H_{N}, N \in L$, then for bounded Borel measurable functions $k_{1}, \ldots, k_{n}$ and any point $y=\sum_{i=1}^{n} \alpha_{i}(T) f_{i} \in H_{N}$, we have

$$
\left(\sum_{i=1}^{n} k_{i}(T) f, \sum_{j=1}^{n} \alpha_{j}(T) f_{j}\right)=\left(f, \sum_{i, j=1}^{n} k_{i}(T) * \alpha_{j}(T) f_{j}\right)=0
$$

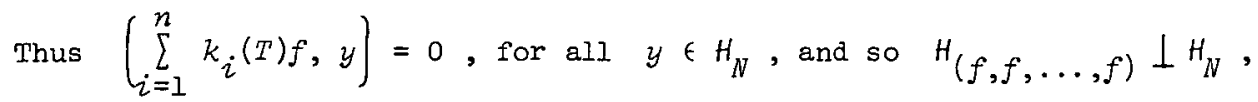
for all $N \in L$, contradicting the maximality of $L$. Hence $H=\sum_{N \in L} H_{N}$. For $f \in H$ we let $f_{N}$ be its component in $H_{N}$, that is

$$
f=\sum_{N \in L} f_{N}, f_{N} \in H_{N}
$$

LEMMA 4. For every Borel function $k$ we have 


$$
D(k(T))=\left\{f \mid f_{N} \in D(k(T)), N \in L ; \sum_{N \in L}\left\|k(T) f_{N}\right\|^{2}<\infty\right\}
$$

and $(k(T) f)_{N}=k(T) f_{N}$.

We first assert that

$$
(D(k(T)))_{N}=H_{N} \cap D(k(T)) .
$$

Clearly $H_{N} \cap D(k(T)) \subset(D(k(T))]_{N} \subset H_{N}$, and so to establish (*) it suffices to show that $(D(k(T)))_{N} \subset D(k(T))$. Let $f \in D(k(T))$; we must show $f_{N} \in D(k(T))$. Now

$$
\left\|E(M) f_{\|}^{2}=\sum_{N}\right\| E(M) f_{N} \|^{2}
$$

and so $\left(E(M) f_{N}, f_{N}\right) \leq(E(M) f, f)$. Thus

$$
\int_{-\infty}^{\infty}|k(t)|^{2}\left(E(d t) f_{N}, f_{N}\right) \leq \int_{-\infty}^{\infty}|k(t)|^{2}(E(d t) f, f)<\infty,
$$

showing that $f_{N} \in D(k(T))$. Thus (*) is proved.

We next show that if $f_{N} \in D(k(T))$, then $k(T) f_{N} \in H_{N}$. Since $f_{N} \in H_{N}$, there exist Borel functions $\alpha_{i}^{r}(t), 1 \leq i \leq n, r \geq 1$, such that

$$
f_{N}=\lim _{r \rightarrow \infty} \sum_{i=1}^{n} \alpha_{i}^{r}(T) f_{i}
$$

Defining $k^{m}(t)$ as before and using the fact that $k^{m}(T)$ is bounded, we see that

$$
\begin{aligned}
k^{m}(T) f_{N} & =\lim \sum_{m \rightarrow \infty}^{n} k_{i=1}^{m}(T) \alpha_{i}^{r}(T) f_{i} \\
& \in H_{N},
\end{aligned}
$$


and since $k^{m}(T) f_{N}+k(T) f_{N}$ as $m \rightarrow \infty, k(T) f_{N} \in H_{N}$. Again, since $k^{m}(T)$ is bounded,

$$
\left\|k^{m}(T) f\right\|^{2}=\sum_{N}\left\|k^{m}(T) f_{N}\right\|^{2}, f \in H
$$

If $f \in D(k(T))$, then by (*) $f_{N} \in D(k(T))$ and we have

$\lim _{m \rightarrow \infty} k^{m}(T) f=k(T) f, \lim _{m \rightarrow \infty} k^{m}(T) f_{N}=k(T) f_{N}$. Thus for any finite set $\pi \subset L$,

$$
\sum_{N \in \pi}\left\|k(T) f_{N}\right\|^{2} \leq\|k(T) f\|^{2}
$$

showing that

$$
\sum_{N \in \pi}\left\|k(T) f_{N}\right\|^{2}<\infty
$$

It will next be shown that $f \in D(k(T))$ provided $f_{N} \in D(k(T))$ and $\sum_{N}\left\|k(T) f_{N}\right\|^{2}<\infty$. Let $\left\{N_{k}\right\} \subset L$ be such that $f_{N}=0$ if $N \notin\left\{N_{k}\right\}$. Thus the terms of the sequence $k(T) f_{N_{k}}$ are orthogonal and since

$$
\sum_{j=1}^{\infty}\left\|k(T) f_{N}\right\|^{2} \leq \sum_{N}\left\|k(T) f_{N}\right\|^{2}<\infty
$$

the series $\sum_{j=1}^{\infty} k(T) f_{N_{j}}$ converges. Now $k(T)$ is a closed operator and thus $f \in D\left(k\left(T^{\prime}\right)\right)$ and

$$
k(T) f=\sum_{j=1}^{\infty} k(T) f_{N}=\sum_{N} k(T) f_{N}
$$

Since $k(T) f_{N} \in H_{N}$, we also have $(k(T) f)_{N}=k(T) f_{N}$. This completes the proof of the lemma.

We shall now adopt the following notation:- 


$$
\begin{gathered}
N=\left\{f_{1}^{N}, f_{2}^{N}, \ldots, f_{n}^{N}\right\}, \mu_{i j}^{N}(\cdot)=\left(E(\cdot) f_{i}^{N}, f_{j}^{N}\right\}, \\
\mu_{N}=\sum_{i=1}^{n} \mu_{i i}^{N}, \mu_{i j}^{N}(M)=\int_{M} m_{i j}^{N}(t) d \mu_{N}(t) .
\end{gathered}
$$

LEMMA 5. $H$ is unitarizy equivalent to $\sum_{N \in L} L^{2}\left(\mu_{i j}^{N}\right)$.

By Lemma 3, $H=\sum_{N \in L} H_{N}$ and by Lemma 2, $H_{N}$ is unitarily equivalent to $L^{2}\left(\mu_{i j}^{N}\right)$, the equivalence being described in that lemma. Combining these results, $H$ is equivalent to $\sum_{N \in L} L^{2}\left(\mu_{i j}^{N}\right)$. Let us suppose $U: H \rightarrow \sum_{N \in L} L^{2}\left(\mu_{i j}^{N}\right)$ is the unitary map obtained above. Note that

$$
\begin{gathered}
\left(U H_{N_{1}}\right)_{N_{2}}=0, N_{1} \neq N_{2} ;=L^{2}\left(\mu_{i j}^{N}\right), i_{1}=N_{2} . \\
\text { For } F=\sum_{N} F_{N} \in \sum_{N} L^{2}\left(\mu_{i j}^{N}\right) \text { let } \\
F_{N}=\left(F_{N 1}, F_{N 2}, \ldots, F_{N n}\right) .
\end{gathered}
$$

LEMMiA 6. For every Borel function $k$ we have $U D(k(T))=$

$$
\left\{\left.F \in \sum_{N} L^{2}\left(\mu_{i j}^{N}\right)\left|\sum_{N} \int_{-\infty}^{\infty}\right| k(t)\right|^{2} \sum_{i, j=1}^{n} m_{i j}(t) F_{N i}(t) \overline{F_{N j}(t)} d \mu_{N}(t)<\infty\right\},
$$

and

$$
(U k(T) f)_{N}(t)=k(t)(U f)_{N}(t)
$$

It is sufficient to prove the second statement since it and Lemma 4 together give the first result. Now

$$
(U k(T) f)_{N}=U(k(T) f)_{N}=U k(T) f_{N} .
$$

Suppose $U f_{N}=\left(\alpha_{1}, \alpha_{2}, \ldots, \alpha_{n}\right) \in L^{2}\left(\mu_{i j}^{N}\right)$. Defining $\alpha_{i}^{m}(t)=\alpha_{i}(t)$ if $\left|\alpha_{i}(t)\right| \leq m,=0$ otherwise and $k^{m}(t)=k(t)$ if $|k(t)| \leq m,=0$ 
otherwise we have

$$
k^{m}(T) f_{N}=\lim _{r \rightarrow \infty} \sum_{i=1}^{n} k^{m}(T) \alpha_{i}^{r}(T) f_{i}^{N}
$$

Thus

$$
\begin{aligned}
U k^{m}(T) f_{N} & =\lim _{m \rightarrow \infty}\left(k^{m}(t) \alpha_{1}^{r}(t), \ldots, k^{m}(t) \alpha_{n}^{r}(t)\right) \\
& =k^{m}(t)\left(\alpha_{1}(t), \ldots, \alpha_{n}(t)\right) .
\end{aligned}
$$

The result follows by letting $m \rightarrow \infty$.

We can now make the following definition.

DEFINITION. Let $T$ be a self adjoint operator on a Hilbert space $H$ and let $\left\{\left(\mu_{i j}^{N}\right)\right\}$ be a family of finite $n \times n$ positive matrix measures defined on the Borel subsets of the line and vanishing on the complement of the spectrum of $T$. Let $U$ be a unitary map between $H$ and $\sum_{N} L^{2}\left(\mu_{i j}^{N}\right)$. The transformation $U$ is an $n \times n$ spectral representation of $H$ onto $\sum_{N} L^{2}\left(\mu_{i j}^{N}\right)$ relative to $T$ if the following conditions are satisfied:-

(a) for every Borel function $k$ defined on the spectrum of $T$ we have

$\operatorname{UD}(k(T))=\left\{\left.F \in \sum_{N} L^{2}\left(\mu_{i j}^{N}\right)\left|\sum_{N} \int_{-\infty}^{\infty}\right| k(t)\right|^{2} \sum_{i, j=1}^{n} F_{N_{i}}(t) \overline{F_{N}(t)} d \mu_{i j}^{N}(t)<\infty\right\} ;$

(b) $\quad(U k(T) f)_{N}(t)=k(t)(U f)_{N}(t)$.

Combining Lemmas $1-6$ we can state:-

THEOREM 1. Every HiZbert space admits an $n \times n$ spectral representation relative to an arbitrary self adjoint operator defined in it, for any value of the integer $n$.

We conclude this section by showing that any $n \times n$ spectral representation may be realized in the fashion described in the lemmas. 
THEOREM 2. Let $U$ be an $n \times n$ spectral representation of $H$ onto $\sum_{K} L^{2}\left(\rho_{i j}^{K}\right)$ relative to a self adjoint operator $T$. Then to each $K$ corresponds a finite set $N \subset H$ such that $\left(\rho_{i j}^{K}\right)=\left(\mu_{i j}^{N}\right), H$ is a direct sum of subspaces $H_{N}$ and $U$ maps $H_{N}$ onto $L^{2}\left(\mu_{i j}^{N}\right)$.

We first note that given an $n \times n$ spectral representation, the resolution of the identity $E$ for $T$ is given by

$$
(U E(M))_{K}(t)=x_{M}(t)(U f)_{K}(t) \in L^{2}\left(\rho_{i j}^{K}\right)
$$

For each $K$ let $\xi^{K l}$ be that element of $\sum_{K} L^{2}\left(\rho_{i j}^{K}\right)$ defined by

$$
\begin{aligned}
\left(\xi^{K 1}\right)_{J}(t) & =(0,0, \ldots, 0), \quad J \neq K, \\
& =(1,0, \ldots, 0), \quad J=K .
\end{aligned}
$$

Let $\xi^{K 2}$ be that element of $\sum_{K} L^{2}\left(\rho_{i j}^{K}\right)$ defined by

$$
\begin{array}{rlrl}
\left(\xi^{K 2}\right)_{J}(t) & =(0,0, \ldots, 0), & J \neq K, \\
& =(0,1,0, \ldots, 0), \quad J=K .
\end{array}
$$

Continuing in this way, we finally let $\xi^{K n}$ be that element of $\sum_{K} L^{2}\left(\rho_{i j}^{K}\right)$ defined by

$$
\begin{array}{rlrl}
\left(\xi^{K n}\right)_{J}(t) & =(0,0, \ldots, 0), & J \neq K, \\
& =(0,0, \ldots, 0, I), \quad J=K .
\end{array}
$$

Note that the $\xi^{K i}$ are elements of $\sum_{K} L^{2}\left(\rho_{i j}^{K}\right)$ since we assumed the measures $\rho_{i i}^{K}$ to be finite. Now define $f_{i}^{N}=U^{-1} \xi^{K i}, i=1,2, \ldots, n$, and set $N=\left\{f_{1}^{N}, \ldots, f_{n}^{N}\right\}$. Then for any Borel set $M \subset R$, 


$$
\begin{aligned}
\rho_{i j}^{K}(M) & =\int_{M} d \rho_{i j}^{K}(t)=\int_{M} \sum_{r, s=1}^{n}\left(\xi^{K i}\right)_{K r}(t) \overline{\left(\xi^{K j}\right)_{K s}}(t) d \rho_{r s}^{K}(t) \\
& \left.=\int U E(M) f_{i}^{N}, U f_{j}^{N}\right)=\left(E(M) f_{i}^{N}, f_{j}^{N}\right) \\
& =\mu_{i j}^{N}(M) .
\end{aligned}
$$

The remaining statements follow from the earlier lemmas.

3.

As in [1] we now give an analytic representation of the unitary map $U$. We shall assume that $T$ is a self adjoint operator in the space $L^{2}(S, \Sigma, \nu)$ where $(S, \Sigma, \nu)$ is a positive measure space. We also assume that there exists an expanding sequence $\left\{S_{n}\right\}$ covering $S$, each element of which has finite measure, and that for bounded sets $M$ the range of $E(M)$ contains only functions which are $v$-essentially bounded on each of the sets $S_{n},(c f .[1], p .1210)$. We shall use the $1 \times 1$ representation theory ([1], Theorem 11, p. 1213) to develop corresponding results for the general case.

We shall require some results from the theory of positive matrix measures and we state them here without proof. Full details may be found in [1, pp. 1341-1342].

LEMMA 7. Let $\left(\mu_{i j}\right)$ be an $n \times n$ positive matrix measure whose elements are continuous with respect to a positive o-finite measure $\mu$. If $\left(m_{i j}\right)$ is the matrix of densities of $\mu_{i j}$ with respect to $\mu$, then there exist non-negative $\mu$-measurable functions $\phi_{i}, \quad 1 \leq i \leq n$, $\mu$-integrable over each bounded interval, and $\mu$-measurable functions $a_{i j}$, $1 \leq i, j \leq n$, such that for $\mu$-almost all $t$

$$
\begin{aligned}
& \text { (a) } \sum_{j=1}^{n} a_{i j}(t) \overline{a_{j k}(t)}=\delta_{i k} \text {, and } \\
& \text { (b) } \sum_{j=1}^{n} \phi_{j}(t) a_{j i}(t) \overline{a_{j k}(t)}=m_{i k}(t) \text {. }
\end{aligned}
$$


LEMMA 8. Let $\left(\mu_{i j}\right),\left(m_{i j}\right), \phi_{i}, a_{i j}, \mu$ be as above. For each $i=1,2, \ldots, n$ let $v_{i}$ be the positive measure defined by

$$
v_{i}(M)=\int_{M} \phi_{i}(t) d \mu(t) \text {. }
$$

Then there exists a unitary map $\Gamma: L^{2}\left(\mu_{i j}\right) \rightarrow \sum_{i=1}^{n} L^{2}\left(\nu_{i}\right) . \Gamma$ is given by

$$
\Gamma\left(E_{1}, F_{2}, \ldots, E_{n}\right)=\left(\sum_{j=1}^{n} a_{1 j} F_{j}, \ldots, \sum_{j=1}^{n} a_{n j} F_{j}\right),
$$

while its inverse is given by

$$
\Gamma^{-1}\left(G_{1}, G_{2}, \ldots, G_{n}\right)=\left(\sum_{j=1}^{n} \overline{a_{1 j} G_{j}}, \ldots, \sum_{j=1}^{n} \bar{a}_{n j} G_{j}\right)
$$

THEOREM 3. Let $(S, \Sigma, \nu)$ be a positive measure space and let $\left\{S_{n}\right\}$ be an expanding sequence of sets of finite measure covering $S$. Let $U$ be an $n \times n$ spectral representation of $L^{2}(S, \Sigma, \nu)$ onto $\sum_{N \in L} L^{2}\left(\mu_{i j}^{N}\right)$ relative to the self adjoint operator $T$ in $L^{2}(S, \Sigma, \nu)$. Let $E$ be the resolution of the identity for $T$ and suppose that for each bounded Borel set $M$ the range of the projection $E(M)$ contains only functions which are $v$-essentially bounded on each of the sets $S_{n}$. Then for each $N \in L$ there are functions $w_{N}^{i}, 1 \leq i \leq n$, defined on the cartesian product of $S$ with the real line and having the properties:

(a) $w_{N}^{i}$ is measurable with respect to the product measure $\nu \times \mu_{N}$;

(b) for each bounded Borel set $M \subset R$ we have

$$
\begin{aligned}
& \underset{s \in S_{n}}{\text { vess } \sup } \int_{M}\left\{\sum_{i, j=1}^{n} m_{i j}^{N}(t) w_{N}^{i}(s, t) \overline{w_{N}^{j}(s, t)}\right\} d \mu_{N}(t)<\infty, n \geq 1 ; \\
& \text { (c) } \left.\left((U f)_{N}(t)\right)=\iint_{S} f(s) w_{N}^{i}(s, t) d v(s)\right]_{i=1}^{n}, f \in L^{2}(S, \Sigma, v),
\end{aligned}
$$


the integrals existing in the mean square sense in $L^{2}\left(\mu_{i j}^{N}\right)$. (See [1], p. 1350 for a discussion of mean square existence of integrals in $\left.L^{2}\left(\mu_{i j}^{N}\right) \cdot\right)$

Let $\phi_{i}^{N}, a_{i j}^{N}, v_{i}^{N}, \Gamma^{N}$ be those functions, measures and unitary maps as described in Lemmas 7 and 8 corresponding to $\left(\mu_{i j}^{N}\right)$. Thus we have

$$
L^{2}(S, \Sigma, \nu) \stackrel{U}{\rightarrow} \sum_{N \in L} L^{2}\left(\mu_{i j}^{N}\right) \stackrel{\Gamma}{\rightarrow} \sum_{N \in L} \sum_{i=1}^{n} L^{2}\left(v_{i}^{N}\right),
$$

where $\Gamma$ is a unitary map constructed from the $\Gamma^{N}$ in an obvious manner. Hence $\Gamma U$ is a unitary map between $L^{2}(S, \Sigma, \nu)$ and $\sum_{N \in L} \sum_{i=1}^{n} L^{2}\left(v_{i}^{N}\right)$. Further, for any Borel function $k$ defined on the spectrum of $T$,

$$
\begin{aligned}
\Gamma U D(k(T)) & =\Gamma\left\{F=\sum F_{N} \mid \sum_{N}\left\|k(t) F_{N}(t)\right\|^{2}<\infty\right\} \\
& =\left\{G=\left.\sum_{N} \sum_{i} G_{N}^{i}\left|\sum_{N} \sum_{i} \int_{-\infty}^{\infty}\right| k(t) G_{N}^{i}(t)\right|^{2} d v_{i}^{N}(t)<\infty\right\} .
\end{aligned}
$$

This result follows readily from the representations of the maps $\Gamma^{N}$ as given in Lemma 8 . That lemma also shows that

$$
(\Gamma U k(T) f)_{N}^{i}(t)=k(t)(\Gamma U f)_{N}^{i}(t) .
$$

Thus $\Gamma U$ is a spectral representation of $L^{2}(S, \Sigma, \nu)$ onto $\sum_{N \in L} \sum_{i=1}^{n} L^{2}\left(v_{i}^{N}\right)$ in the sense of $[1, p .1208],(-a 1 \times 1$ spectral representation in terms of our definition). Applying [1, Theorem 11 , p. 1213], we see that there exist measurable functions $W_{N}(s, t)$ defined on $S \times R$ such that for each bounded Borel set $M \subset R$

$$
\underset{s \in S_{n}}{v \text {-ess } \sup _{M}}\left|\int_{N_{i}}(s, t)\right|^{2} d v_{i}^{N}(t)<\infty, n \geq 1,
$$


and

$$
(\Gamma \cup f)_{N}^{i}(t)=\int_{S} f(s) \overline{W_{N_{i}}(s, t)} d \nu(s), f \in L^{2}(S, \Sigma, \nu)
$$

the integral existing in the mean square sense in $L^{2}\left(v_{i}^{N}\right)$. Now for $N \in L, 1 \leq i \leq n$, define $w_{N}^{i}$ by

$$
w_{N}^{i}(s, t)=\sum_{j=1}^{n} \frac{.}{a_{i j}(t) w_{N}(s, t)}
$$

Clearly each $w_{N}^{i}$ is measurable, and for $n \geq 1$

$$
\begin{aligned}
& \underset{s \in S_{n}}{\nu-e s s} \sup \int_{M j, k=1} \sum_{j k}^{n}(t) \overline{w_{N}^{j}}(s, t) \overline{W_{N}^{k}(s, t)} d \mu_{N}(t) \\
& =\underset{s \in S_{n}}{v-\operatorname{ess} \sup } \int_{M i, j, k=1} \sum_{i}^{n} \phi_{i j}^{N}(t) \overline{a_{i k}(t)} w_{N}^{j}(s, t) \overline{W_{N}^{k}(s, t)} d \mu_{N}(t) \\
& =\underset{s \in S_{n}}{v-e s s} \sup \sum_{i=1}^{n} \int_{M} \sum_{j, k, p, q=1}^{n} a_{i j}(t) \overline{\alpha_{i k}(t) a_{j p}(t) a_{k q}}(t) \overline{W_{N p}(s, t)} \\
& \text { - } W_{N q}(s, t) d v_{i}^{N}(t) \\
& =\underset{s \in S_{n}}{v-e s s} \sup \sum_{i=1}^{n} \int_{M}\left|w_{N i}(s, t)\right|^{2} d v_{i}^{N}(t) \\
& \leq \sum_{i=1}^{n} v-\operatorname{ess} \sup \int_{M}\left|w_{N i}(s, t)\right|^{2} d \nu_{i}^{N}(t)<\infty
\end{aligned}
$$

Finally, for $f \in L^{2}(S, \Sigma, \nu)$,

$$
(\Gamma U f)_{i}^{N}(t)=\lim _{p \rightarrow \infty} \int_{S_{p}} f(s) \overline{W_{N i}(s, t)} d v(s),
$$

the limit being taken in the topology of $L^{2}\left(U_{i}^{N}\right)$. Applying $\Gamma^{-1}$ we have 


$$
\begin{aligned}
\left((U f)_{N}(t)\right) & =\lim _{r \rightarrow \infty}\left(\sum_{j=1}^{n} \overline{a_{i j}(t)} \int_{S_{r}} f(s) \overline{W_{N j}(s, t)} d v(s)\right)_{i=1}^{n} \\
& =\lim _{r \rightarrow \infty}\left(\int_{S_{p}} f(s) w_{N}^{i}(s, t) d v(s)\right)_{i=1}^{n} .
\end{aligned}
$$

This completes the proof of the theorem.

THEOREM 4. With the notation of the previous theorem we have

$$
f(s)=\sum_{N \in L} \int_{-\infty}^{\infty} \sum_{i, j=1}^{n} m_{i j}(t)\left(U f_{N}\right)_{i}(t) \overline{w_{N}^{j}(s, t)} d \mu_{N}(t),
$$

$f \in L^{2}(S, \Sigma, \nu)$, the integrals existing mean square in $L^{2}(S, \Sigma, v)$ and the series converging in the norm of $L^{2}(S, \Sigma, \nu)$.

$$
\text { For } f \in L^{2}(S, \Sigma, v), f=\sum_{N} f_{N}=\sum_{N} \sum_{i} f_{N}^{i} \text { where } f_{N}^{i} \text { is the }
$$

component of $f$ in $(\Gamma U)^{-1} L^{2}\left(v_{i}^{N}\right)$. Further by [1, Corollary to Theorem 11, p. 1213],

$$
\begin{aligned}
f_{N} & =\sum_{i=1}^{n} f_{N}^{i}=\sum_{i=1}^{n} \lim _{r \rightarrow \infty} \int_{-r}^{r}(\Gamma U f)_{N}^{i}(t) W_{N i}(s, t) d v_{i}^{N}(t) \\
& =\lim _{r \rightarrow \infty} \int_{-r}^{r} \sum_{i, j=1}^{n}\left(U f_{N}\right)_{j}(t) a_{i j}(t) w_{N i}(s, t) \phi_{i}(t) d \mu_{N}(t) \\
& =\lim _{r \rightarrow \infty} \int_{-r}^{r} \sum_{i, j=1}^{n}\left(U f_{N}\right)_{j}(t) a_{i j}(t) \phi_{i}(t) \sum_{k=1}^{n} W_{N k}(s, t) \sum_{p=1}^{n} \overline{a_{i p}(t) a_{p k}}(t) d \mu_{N}(t) \\
& =\lim _{r \rightarrow \infty} \int_{-r}^{r} \sum_{j, p=1}^{n}\left(U f_{N}\right)_{j}(t) \sum_{i=1}^{n} \phi_{i}(t) a_{i j}(t) \overline{a_{i p}(t)} \sum_{k=1}^{n} a_{p k}(t) W_{N k}(s, t) d \mu_{N}(t) \\
& =\lim _{r \rightarrow \infty} \int_{-r}^{r} \sum_{j, p=1}^{n} m_{j p}(t)\left(U f_{N}\right)_{j}(t) \overline{w_{N}^{p}(s, t) d \mu_{N}(t) .}
\end{aligned}
$$

From this, the result follows immediately. 


\section{Reference}

[1] Nelson Dunford and Jacob T. Schwartz, Linear operators, Part II: Spectral theory. Self adjoint operators in Hilbert space (Interscience [John Wiley \& Sons], New York, London, 1963).

University of Toronto, Toronto, Canada. 